\title{
ON RINGS WITH ONE-SIDED FIELD OF QUOTIENTS
}

\author{
ENZO R. GENTILE ${ }^{1}$
}

This note extends certain results of Cartan-Eilenberg's Homological algebra, Chapter VII, Integral domains, to rings with one-sided field of quotients. Let $R$ be a ring with a left field of quotients $Q$. We prove the following results. If $A$ is a left $R$-module and $t A$ the set of all its torsion elements then $\operatorname{Tor}_{1}^{R}(Q / R, A)=t A$. With a slightly modified definition of inversible ideal it is proved that the notions of projective and inversible ideals are equivalent. If, in addition, $R$ is left semihereditary then every finitely generated torsion-free right $R$-module is $R$-flat. Every finitely generated torsion-free left $R$-module can be imbedded in a finitely generated free left $R$-module if and only if $R$ has both left and right field of quotients. Finally we give an example of a ring having a left field of quotients but not a right one. This implies that there exist finitely generated torsion-free left $R$-modules which cannot be imbedded in a projective left $R$-module.

1. Generalities. Our basic reference will be [1]. Let $R$ be a ring with identity and without zero divisors. $R$ is said to have a left field of quotients (see [2, Chapter I, $\S 9$, Exercises 8 and 9]) if $R$ is a subring of a division ring $Q$ in which every element is a "left quotient" of elements in $R$, that is, every element in $Q$ can be written as $x^{-1} \cdot y$, where $x \in R, y \in R$ and $x \neq 0$. The following conditions for $R$ to be a ring with a left field of quotients are equivalent:

G1. For every pair $\left(x, x^{\prime}\right) \in R \times R, x^{\prime} \neq 0$, there exists a pair $(u, v)$ $\in R \times R, u \neq 0$ such that $u \cdot x=v \cdot x^{\prime}$.

G2. The intersection of any two nonzero left ideals of $R$ is different from zero.

G3. There exists a division ring $Q$ containing $R$ such that for every finite set $\left\{q_{1}, \cdots, q_{n}\right\}$ of elements in $Q$, there exists an element $r \in R, r \neq 0$ such that $r \cdot q_{i} \in R$ for $i=1, \cdots, n$.

As in [1], in any module $A$ we can consider the set of torsion elements $t A$ and the set of divisible elements $\delta A$. If $R$ has a left field of quotients and $A$ is a left (resp. right) $R$-module then $t A$ (resp. $\delta A$ ) is a submodule of $A$. This follows immediately from $\mathrm{G} 1$.

Proposition 1.1. A torsion-free left $R$-module is injective if and only if it is divisible.

Received by the editors August 13, 1959.

1 The author is a Visiting Fellow at Princeton University holding a Fellowship from the Consejo de Investigaciones Cientificas, Argentina. 
Proof. The necessity is identical to the necessity of Proposition $1.2,[1$, Chapter VII]. To prove the sufficiency assume that $A$ is a torsion-free and divisible left $R$-module. Consider a homomorphism $f: I \rightarrow A$ of a left ideal $I$ of $R$ into $A$. For every $i \in I, i \neq 0$ there exists a unique $a_{i} \in A$ such that $f i=i \cdot a_{i}$. Let $j$ be any nonzero element of $I$. Let $u$ and $v, u \neq 0$ be elements of $R$ satisfying $u \cdot i=v \cdot j$. Then we have

$$
u \cdot i \cdot a_{i}=u \cdot f i=f(u \cdot i)=f(v \cdot j)=v \cdot f j=v \cdot j \cdot a_{j}=u \cdot i \cdot a_{j}
$$

and therefore $a_{i}=a_{j}=a$. Then for every $j \in I, f j=j \cdot a$ and therefore $A$ is injective.

2. The field of quotients. Let $Q$ be the left field of quotients of $R$. We introduce in $R$ the following partial order. (1) For every $x, 0<x,(2)$ if $x \neq 0$ and $y \neq 0 x<y$ if and only if $x^{-1} \cdot R \subset y^{-1} \cdot R .[R,<]$ is a directed set. In fact, let $x$ and $y$ be any two nonzero elements of $R$. There exist two elements $u, v$ in $R u \neq 0$ and $v \neq 0$ such that $u \cdot x=v \cdot y$. Then

$x^{-1} \cdot R \subset x^{-1} \cdot u^{-1} \cdot R=(u \cdot x)^{-1} \cdot R$ and $y^{-1} \cdot R \subset y^{-1} \cdot v^{-1} \cdot R=(v \cdot y)^{-1} \cdot R$ and thus $x<u \cdot x$ and $y<u \cdot x$.

Let $R_{x}=x^{-1} \cdot R$ if $x \neq 0$ and $R_{0}=0$. We define $f_{x y}: R_{x} \rightarrow R_{y}$ for every pair $x<y$, by inclusion. Then $\left\{R_{x}, f_{y z}\right\}$ becomes a directed system of right $R$-modules and it is obvious that the direct limit is $Q$. Since each $R_{x}$ is $R$-projective we have

Proposition 2.1. $Q$ is a right flat $R$-module.

From the exact sequence $0 \rightarrow R \rightarrow Q \rightarrow Q / R \rightarrow 0$ and for every left $R$-module $A$ we have the exact sequence

$$
0 \rightarrow \operatorname{Tor}_{1}(Q / R, A) \rightarrow A \rightarrow Q \otimes A \rightarrow Q / R \otimes A \rightarrow 0 \quad\left(\otimes=\otimes_{R}\right) .
$$

Now by using G3 one can prove

Proposition 2.2. $\operatorname{Tor}_{1}^{R}(Q / R, A)=t A$.

Proof. The same as in the commutative case. See [2, Chapter III §2, Theorem 2 and also Exercise §2-7g].

Then if $A$ is a torsion-free left $R$-module we have the exact sequence

$$
0 \rightarrow A \rightarrow Q \otimes A \rightarrow Q / R \otimes A \rightarrow 0 .
$$

Observe that since $Q \otimes A$ is torsion-free and divisible it is $R$-injective. Applying cohomology to (1) we get the isomorphism

$$
\operatorname{Hom}_{R}\left(A^{\prime}, K \otimes A\right) \approx \operatorname{Ext}_{R}^{1}\left(A^{\prime}, A\right) \quad(K=Q / R),
$$

where $A^{\prime}$ is a torsion module (i.e. $t A^{\prime}=A^{\prime}$ ) and $A$ is torsion-free.

Now we recall 
G4. $R$ has a left field of quotients if and only if for every nonzero left ideal $I$ of $R, R / I$ is a torsion module.

Then G4 and (2) give the isomorphism

$$
\operatorname{Hom}_{R}(R / I, K) \approx \operatorname{Ext}_{R}^{1}(R / I, R) .
$$

$\operatorname{Ext}_{R}^{n}(R / I, K), n>1$ can be computed by using the exact sequences

$$
\begin{aligned}
& 0 \rightarrow I \rightarrow R \rightarrow R / I \rightarrow 0, \\
& 0 \rightarrow R \rightarrow Q \rightarrow K \rightarrow 0,
\end{aligned}
$$

the fact that $Q$ is $R$-injective, and Theorem 1.5 [1, Chapter VI].

3. Inversible ideals. A left ideal $I$ of $R^{*}$ is called inversible if there exist elements $q_{1}, \cdots, q_{n}$ in $Q$ and $a_{1}, \cdots, a_{n}$ in $I$ such that $I \cdot q_{i} \subset R$ and $\sum_{i} q_{i} \cdot a_{i}=1, i=1, \cdots, n$.

Proposition 3.1. In order that a nonzero ideal $I$ of $R$ be projective it is necessary and sufficient that $I$ be an inversible ideal.

Proof. The sufficiency is the same as Proposition $3.2[1$, Chapter VII]. Let $I$ be a projective left ideal and let $\left\{a_{i}\right\},\left\{\phi_{i}\right\}$ be families of elements of $I$ and $R$-maps $\phi_{i}: I \rightarrow R$ respectively, satisfying $a=\sum_{i} \phi_{i}(a) \cdot a_{i}$ for every $a \in I$. Let $x \neq 0, x \in I$. We define $q_{i} \in Q$ by $q_{i}=x^{-1} \cdot \phi_{i}(x)$. Let $y$ be any element of $I$. There exist $u$ and $v$ in $R$, $v \neq 0$ such that $u \cdot x=v \cdot y$. Then $v \cdot \phi_{i}(y)=\phi_{i}(v \cdot y)=\phi_{i}(u \cdot x)=u \cdot \phi_{i}(x)$ $=u \cdot x \cdot q_{i}=v \cdot y \cdot q_{i}$ and therefore $\phi_{i}(y)=y \cdot q_{i}$ for every $y \in I$ and furthermore $I \cdot q_{i} \subset R$ for every $i$.

Since $\phi_{i}(x)=x \cdot q_{i}$ it follows that $q_{i}=0$ for almost every $i$. Finally $x=\sum_{i}\left(\phi_{i} x\right) \cdot a_{i}=\sum_{i} x \cdot q_{i} \cdot a_{i}=x \sum_{i} q_{i} \cdot a_{i}$ implies $\sum_{i} q_{i} \cdot a_{i}=1$.

By using the same proof as Proposition 3.3 and Proposition 3.4 [1, Chapter VII] we have

Corollary 3.1. Any inversible ideal is finitely generated.

Corollary 3.2. If $I$ is inversible and $A$ divisible then $\operatorname{Ext}_{R}^{1}(R / I, A)$ $=0$.

This last corollary implies

COROllary 3.3. If, in addition, $R$ is left hereditary a left $R$-module is divisible if and only if it is injective.

By using Corollary 3.1 we can prove

Proposition 3.2. Let $R$ be a left hereditary ring. Then every torsionfree right $R$-module $C$ is flat. 
Proof. By Corollary $3.1 R$ is noetherian. Then using the Proposition 5.5 [1, Chapter VI] we have the monomorphism

$$
\operatorname{Tor}_{1}^{R}(C, R / I) \rightarrow \operatorname{Hom}_{R}\left(\operatorname{Ext}_{R}^{1}(R / I, R), C\right) .
$$

(3) in $\$ 2$ gives the monomorphism

$$
\operatorname{Tor}_{1}^{R}(C, R / I) \rightarrow \operatorname{Hom}_{R}\left(\operatorname{Hom}_{R}(R / I, K), C\right)
$$

where $C$ is a torsion-free right $R$-module. Since $\operatorname{Hom}(R / I, K)$ is a torsion module the second part of (1) is zero and hence $\operatorname{Tor}_{1}^{R}(C, R / I)$ $=0$ for every $I$, which means that $C$ is right $R$-flat.

Note. It can be seen that the monomorphism of Proposition 5.5, [1, Chapter VI] also holds assuming that $R$ is left semihereditary and $A$ is finitely generated and with a projective resolution composed of finitely generated modules. Since this is the case if $A=R / I$ for $I$ finitely generated, it follows by using the argument of the last proposition that $\operatorname{Tor}_{1}^{R}(C, R / I)=0$ for $I$ finitely generated. Now, by taking direct limit this holds for every left ideal $I$. Then our last proposition can be stated for left semihereditary rings and this gives the generalization of Proposition 4.2 [1, Chapter VII].

4. Imbedding in a free module. We denote by $(F)$ the following property:

(F) Every finitely generated torsion-free left $R$-module can be imbedded in a finitely generated free left $R$-module.

Proposition 4.1. $R$ satisfies (F) if and only if in addition $R$ has a right field of quotients.

Proof. If $R$ satisfies (F) let $x$ and $y$ be any two nonzero elements of $R$. Let $A=R \cdot x^{-1}+R \cdot y^{-1}$ be the left $R$-submodule of $Q$ generated by $x^{-1}$ and $y^{-1}$. Let $F$ be a finitely generated free left $R$-module containing $A$. Let $f_{1}, \cdots, f_{n}$ be a basis of $F$. Then $A$ (and a fortiori $F$ ) contains an element $e=x \cdot x^{-1}=y \cdot y^{-1}$. Let $x^{-1}=\sum_{i} r_{i} \cdot f_{i}$ and $y^{-1}$ $=\sum_{i} s_{i} \cdot f_{i}$ with $r_{i} \in R, s_{i} \in R$ and $r_{1} \neq 0$ and $s_{1} \neq 0$. Then $e=\sum_{i} x \cdot r_{i} \cdot f_{i}$ $=\sum_{i} y \cdot s_{i} \cdot f_{i}$ and this implies $x \cdot r_{1}=y \cdot s_{1}$ which means that that $R$ has a right field of quotients.

If $R$ has a right field of quotients let $A$ be a finitely generated torsion free left $R$-module. Then $A$ can be imbedded in $Q \otimes A$, which is a left $Q$-vector space. If $a_{1}, \cdots, a_{n}$ generate $A$ then $1 \otimes a_{1}, \cdots, 1 \otimes a_{n}$ generate $Q \otimes A$ as a $Q$-vector space. Let $e_{1}, \cdots, e_{m}$ be a basis of $Q \otimes A$. Then for every $i=1, \cdots, n$

$$
a_{i}=\sum_{j} q_{i j} \cdot e_{j}, \quad q_{i j} \in Q .
$$

By the dual of G3 there exists $r \in R, r \neq 0$ such that $q_{i j} \cdot r \in R$ for every 
pair $i j$. Then $a_{i}=\sum_{j}\left(q_{i j} r\right)\left(r^{-1} \cdot e_{j}\right)$ and therefore $A$ is contained in the free left $R$-module generated by $r^{-1} \cdot e_{1}, \cdots, r^{-1} \cdot e_{m}$.

In the next section we will give an example of a ring having a left field of quotients but not a right one. This example and Proposition 4.1 imply that there exist torsion-free finitely generated left modules which cannot be imbedded in a projective left $R$-module.

It is easy to see that every ring without zero divisors in which every left ideal is principal is a ring with a left field of quotients. Then if $R$ is a left and right principal ideal ring every finitely generated torsion-free left (or right) $R$-module is free.

Proposition 4.2. Let $R$ be a left semihereditary ring with a left field of quotients. Then $R$ satisfies the condition (F) stated for right ideals.

Proof. Let $A$ be a finitely generated torsion free right $R$-module. Then $A$ is $R$-flat, therefore $\operatorname{Tor}_{1}^{R}(A, Q / R)=0$ and $A$ can be imbedded in $A \otimes Q$. Now the proof is the same as the second part of Proposition 4.1.

5. An example. We will give in this section an example of a ring having a left field of quotients but not a right one. Let $K$ be a commutative field and $f: K \rightarrow K^{\prime}$ an isomorphism of $K$ onto a subfield $K^{\prime}$ of $K$ satisfying $\left[K: K^{\prime}\right]>1$. Let $R=K[X, f]$ be the ring of (twisted) polynomials $\sum r_{i} \cdot X^{i}$ with the ordinary sum and with the following product:

$$
\left(r \cdot X^{i}\right) \circ\left(s \cdot X^{j}\right)=r \cdot f^{i}(s) \cdot X^{i+j} .
$$

It is easy to see that $[R, 0]$ is a ring (see [2, Chapter IV, Exercise, $\S 5,10])$. The verification that $R$ satisfies $\mathrm{G} 1$ is immediate and then $R$ has a left field of quotients. In order to prove that $R$ has no right field of quotients we must observe that if $E$ is any $K^{\prime}$-vector space contained in $K$ the set (par abus de langage) denoted by $E[X]$ of all the polynomials of $R$ with coefficients in $E$ is a right ideal of $R$. Since $\left[K: K^{\prime}\right]>1$ we can find two nonzero vector spaces $E$ and $E^{\prime}$ over $K^{\prime}$ satisfying $E \cap E^{\prime}=0$. But this implies $E[X] \cap E^{\prime}[X]=0$ and so $R$ does not satisfy the dual of G2 and therefore has not a right field of quotients.

\section{REFERENCES}

1. H. Cartan and S. Eilenberg, Homological algebra, Princeton University Press, 1956.

2. N. Bourbaki, Algèbre, Livre II, Chapitres I (1942), II (1948), III (1952), Paris, Hermann.

RUTGERS UNIVERSITY AND

Princeton UnIVERSITY 